\title{
Enfisema subcutáneo extenso
}

Extensive subcutaneous emphysema

Enfisema subcutâneo extenso

Carlos Alberto Peña Pérez, ${ }^{*}$ Raúl Carrillo Esper,‡ José Martín Meza Márquez, ${ }^{\star}$

Yesica Ivone Martínez Baltazar*

\section{RESUMEN}

El enfisema subcutáneo es una complicación relativamente frecuente y por lo general autolimitada tras la colocación de un tubo de toracostomía. El enfisema subcutáneo relacionado con la ventilación mecánica a presión positiva puede condicionar tensión subcutánea palpable (aumento de volumen y crepitación subcutánea), cierre palpebral, disfagia, disfonía y, asociado con pneumoperitoneo, compromiso de la vía aérea e insuficiencia respiratoria «fenómeno de tensión» clasificándolo por estas características como enfisema subcutáneo extenso.

Palabras clave: Enfisema subcutáneo, ventilación mecánica, infección, clasificación, manejo.

\section{ABSTRACT}

Subcutaneous emphysema is common complication self-limited complication of tube thoracostomy. Any subcutaneous emphysema on positive pressure ventilation, causing palpable cutaneous tension, palpebral closure, dysphagia, and dysphonia or associated with pneumoperitoneum, airway compromise, "tension phenomenon" and respiratory failure is labelled extensive subcutaneous emphysema.

Keywords: Subcutaneous emphysema, positive pressure ventilation, infection, classification, management.

\section{RESUMO}

O enfisema subcutâneo é uma complicação relativamente comum e geralmente autolimitada após a colocação de um tubo de toracostomia. O enfisema subcutâneo relacionado à ventilação mecânica com pressão positiva pode levar a tensão subcutânea palpável (aumento de volume e crepitação subcutânea), fechamento da pálpebra, disfagia, disfonia; associado a pneumoperitônio, comprometimento das vias aéreas e insuficiência respiratória «fenômeno tensional» classificando-o por essas características como enfisema subcutâneo extenso.

Palavras-chave: Enfisema subcutâneo, ventilação mecânica, infecção, classificação, manejo.

\section{INTRODUCCIÓN}

El enfisema subcutáneo es una complicación relativamente frecuente y por lo general autolimitada tras la colocación de un tubo de toracostomía. El enfisema subcutáneo relacionado con la ventilación mecánica a presión positiva puede condicionar tensión subcutánea palpable (aumento de volumen y crepitación subcutánea), cierre palpebral, disfagia, disfonía y, asociado con pneumoperitoneo, compromiso de la vía aérea e insuficiencia respiratoria «fenómeno de tensión» clasificándolo por estas características como enfisema subcutáneo extenso.

\footnotetext{
* Centro Médico Naval, Secretaría de Marina Armada de México.

₹ Instituto Nacional de Rehabilitación.
}

Recibido: 04/03/2020. Aceptado: 23/12/2020.

Citar como: Peña PCA, Carrillo ER, Meza MJM, Martínez BYI. Enfisema subcutáneo extenso. Med Crit. 2021;35(2):101-105. https://dx.doi.org/10.35366/99531

www.medigraphic.com/medicinacritica

\section{PRESENTACIÓN DEL CASO}

El caso que se presenta es el de un hombre de 42 años de edad, hospitalizado en la Unidad de Terapia Intensiva de Adultos (UTIA) por choque séptico y disfunción orgánica múltiple secundario a un absceso profundo de cuello ya drenado quirúrgicamente y con desarrollo de Klebsiella pneumoniae en cultivos del material purulento obtenido del absceso, hemocultivo, urocultivo y cultivo de secreción bronquial. El manejo brindado en la UTIA fue con base en paquetes, recomendaciones y metas de la campaña para incrementar la supervivencia en sepsis, con soporte multiorgánico, hemodiálisis intermitente por lesión renal aguda y ventilación mecánica invasiva por síndrome de insuficiencia respiratoria aguda grave (Figura 1). En su día siete de estancia en la UTIA con soporte mecánico ventilatorio invasivo en modo controlado con fracción inspirada de oxígeno $\left(\mathrm{FiO}_{2}\right)$ al $60 \%$, presión positiva al final de la espiración (PEEP) $10 \mathrm{cmH}_{2} \mathrm{O}$, frecuencia respiratoria de 18/minuto, presión control (PC) de $20 \mathrm{cmH}_{2} \mathrm{O}$, presentaba con estos parámetros una saturación por oximetría de pulso de $91 \%$ y relación $\mathrm{PaO}_{2} / \mathrm{FiO}_{2} 160 \mathrm{mmHg}$. Desarrolló súbitamente incremento en las presiones de la vía aérea con desaturación progresiva, inestabilidad hemodinámica y aumento de volumen en el tórax anterior bilateral, presencia de crepitación a la palpación del tórax y abdomen, y disminución de ruidos respiratorios en ambos campos pulmonares a la auscultación. En rastreo ultrasonográfico del tórax con signo de «código de barras» en ambos hemitórax, por lo que se colocó de manera inmediata sonda endopleural en el hemitórax derecho percibiendo fuga aérea inmediata con mejoría parcial de la oxigenación por oximetría de pulso $\left(\mathrm{SpO}_{2}\right.$ $75 \%$ ) e incremento en los parámetros de presión arterial media (PAM $50 \mathrm{mmHg}$ ). En la radiografía simple portátil del tórax con imagen de pneumotórax bilateral con reexpansión parcial del parénquima pulmonar derecho y pnemotórax izquierdo, por lo que se colocó otra sonda endopleural en el hemitórax izquierdo mejorando tras dicha intervención la $\mathrm{SpO}_{2}$ al $96 \%$ y la TAM a 70 $\mathrm{mmHg}$. En control radiográfico simple, con reexpansión del parénquima pulmonar bilateral e imagen en relación enfisema subcutáneo extenso (grado V) (Figura 2 A-C), por lo que se decidió realizar incisiones cutáneas infraclaviculares con disección roma subcutánea hasta la 
fascia pectoral mayor bilateral y colocación de drenajes de tipo BioVac, con lo cual se logró la resolución del enfisema subcutáneo en 24 horas (Figura 3). Se retiraron los drenajes BioVac 48 horas posteriores a su colocación sin recurrencia del enfisema subcutáneo.

\section{DISCUSIÓN}

El enfisema subcutáneo ocurre cuando el aire entra por debajo de la piel y entre los tejidos blandos. Esto suele ocurrir en los tejidos blandos de la pared torácica o el cuello, pero también puede extenderse a otras partes

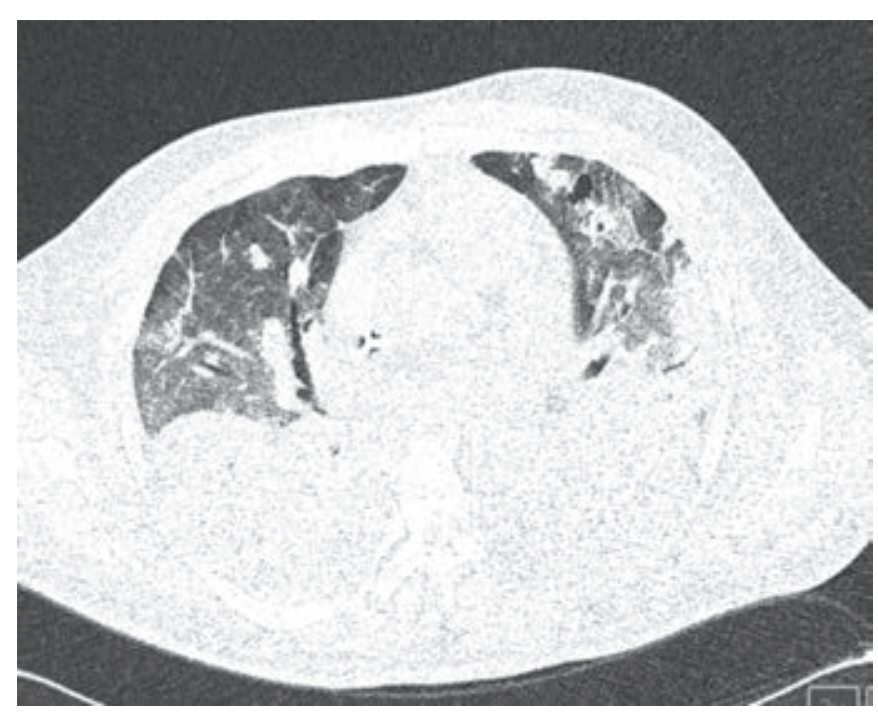

Figura 1: Tomografía axial computarizada (ventana para pulmón) donde se observa imagen en vidrio esmerilado y múltiples áreas de consolidación pulmonar predominantemente en áreas declive bilateral. del cuerpo. ${ }^{1}$ Puede surgir como resultado de una serie de procesos incluyendo el trauma contuso o penetrante, pneumotórax por barotrauma, infección, malignidad, o como complicación de procedimientos quirúrgicos, e inclusive de forma espontánea. ${ }^{2}$ El signo más común visible y el síntoma del enfisema subcutáneo es el aumento de volumen alrededor del cuello acompañado de dolor torácico. Otros signos y síntomas incluyen dolor en la garganta, dolor en el cuello, dificultad para deglutir, dificultad para respirar, sibilancias y distensión. ${ }^{2}$ Por lo tanto, a menudo causa síntomas mínimos, no es peligroso en sí mismo y no requiere de un tratamiento específico. ${ }^{2}$ Sin embargo, si compromete los tejidos más profundos de la salida torácica, el pecho y la pared abdominal, será una condición grave y estresante que amenaza la vida. Se llega a complicar por restricción de la reexpansión pulmonar completa y conducir a una elevación en la presión de la vía respiratoria, condicionar acidosis respiratoria grave, fracaso ventilatorio, disfunción del marcapasos y "fenómeno de tensión». ${ }^{2}$ El diagnóstico se realiza mediante examen físico (crepitación a la palpación sobre el área afectada) y estudio radiológico simple que evidencia la presencia de aire sobre el área afectada. ${ }^{1} \mathrm{El}$ enfisema subcutáneo a menudo representa un dilema de manejo. Éste debe comenzar con un esfuerzo concertado para identificar la causa ofensiva de la disección subcutánea del aire. ${ }^{3}$ Se han descrito varios abordajes, incluyendo el uso de incisiones cutáneas, agujas y drenajes subcutáneos, y la mediastinotomía cervical. ${ }^{3-6}$

Por lo general, el enfisema subcutáneo es secundario a un desgarro en el tejido pulmonar. Después de la ruptura alveolar, el aire se mueve preferentemente desde le intersticio pulmonar a lo largo de las vainas
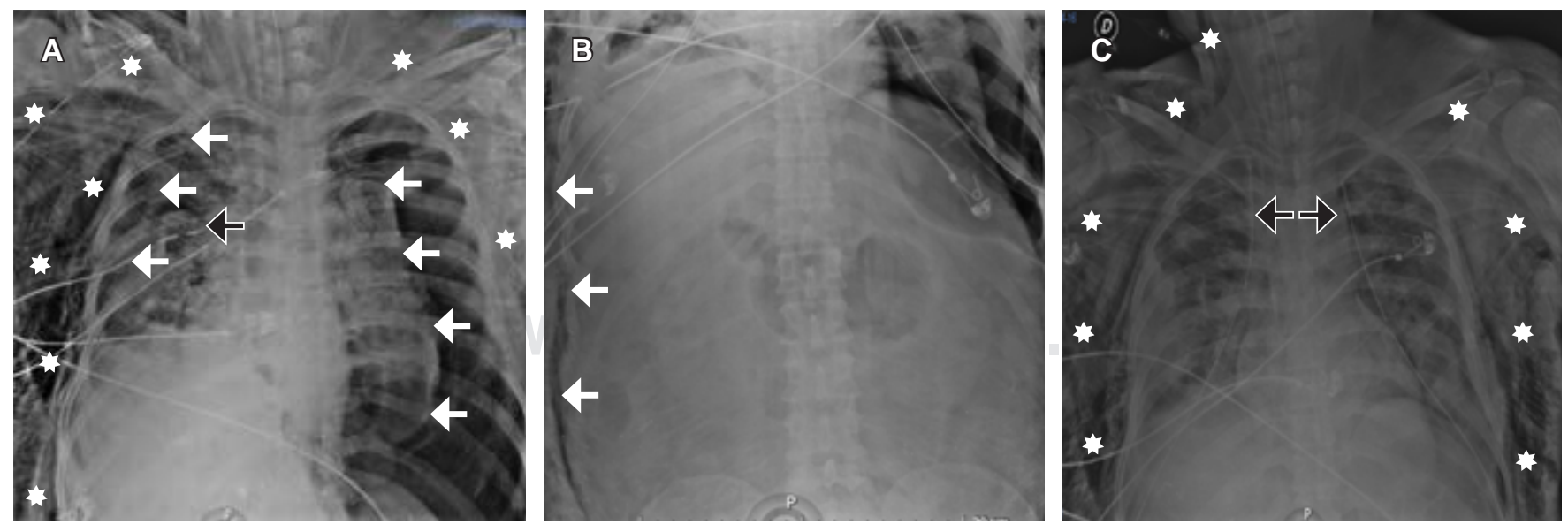

Figura 2: Secuencia de radiografías simples (portátiles) de tórax. A) Flechas blancas delimitan borde de parénquima pulmonar colapsado; asteriscos blancos demarcan la distribución de imagen radiolúcida secundaria a enfisema subcutáneo; flecha negra: señala la punta de sonda endopleural en hemitórax derecho. B) Flechas blancas demarcan la imagen radiolúcida secundaria a enfisema subcutáneo en el trayecto de toda la pared abdominal. C) Flechas negras señalan la presencia de sonda endopleural en ambos hemitórax con adecuada reexpansión del parénquima pulmonar; asteriscos blancos señalan la distribución de imagen radiolúcida secundaria a enfisema subcutáneo. 
broncovasculares hasta el hilio pulmonar desde donde puede pasar superficialmente hasta la fascia endobronquial hacia la entrada torácica. ${ }^{7}$ Por lo tanto, el mejor sitio para la descompresión del enfisema subcutáneo es a nivel de la entrada torácica. En los pacientes con tubo de toracostomía para la corrección de pneumotórax, el enfisema subcutáneo se puede desarrollar siempre que la pleura parietal se rompa, creando una vía para el paso del aire hacia el tejido subcutáneo. ${ }^{8}$

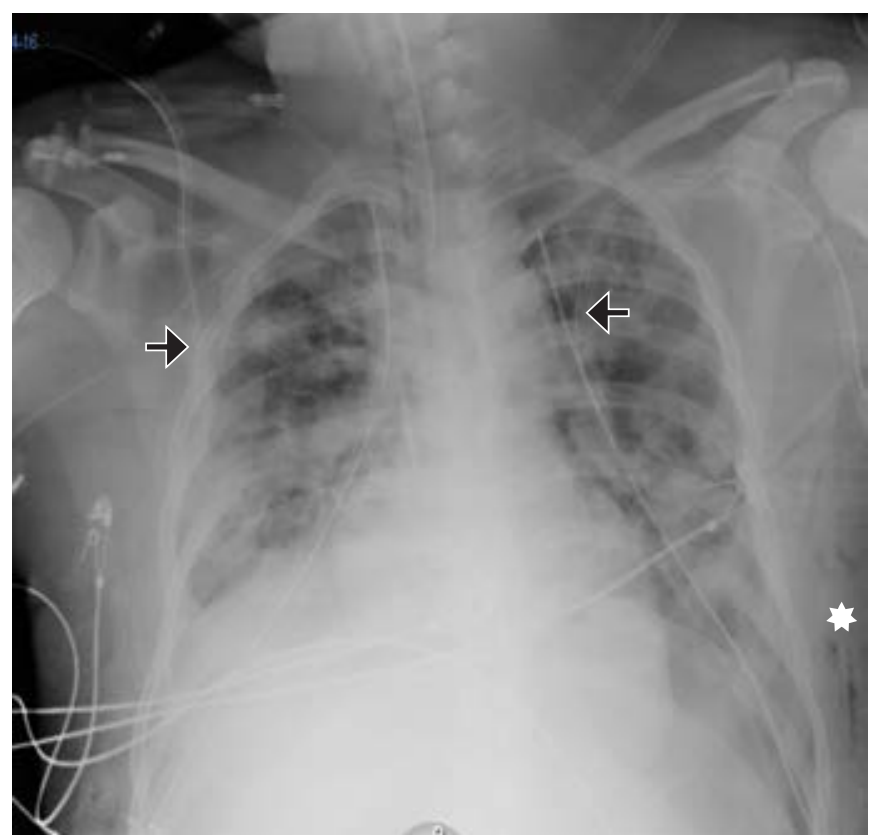

Figura 3: Radiografía simple (portátil) de tórax. Flechas negras señalan la presencia de drenajes subcutáneos de tipo BioVac en área infraclavicular y subcutánea. Se aprecia estatus de sonda endopleural en ambos hemitórax con adecuada reexpansión del parénquima pulmonar. Asterisco blanco señala el área limitada de imagen radiolúcida secundaria a enfisema secundario 24 horas posteriores a la colocación de los drenajes subcutáneos tipo BioVac.
A la fecha, la única clasificación descrita para estimar la severidad del enfisema subcutáneo es la publicada por Aghajanzadeh ${ }^{9}$ en 2015 , donde lo estratifica en cinco grados con base en la extensión y áreas afectadas: 1) circunscrito sólo a la base del cuello, 2) todo el cueIlo, 3) pectoral mayor, 4) tórax y cuello, 5) tórax, cuello, cara, piel cabelluda, extremidades superiores, pared abdominal y escroto (Figura 4).

Se han intentado varios métodos para controlar el enfisema subcutáneo extenso y debilitante. En la revisión más completa de la literatura hasta la fecha, Johnson determinó que no se puede considerar ningún método como el mejor en ausencia de ensayos clínicos aleatorizados. Las incisiones infraclaviculares, los drenajes subcutáneos y el aumento de la succión en un drenaje torácico in situ proporcionan un alivio adecuado. Seleccionar cualquier método depende de factores inherentes al paciente y de las preferencias del médico a cargo. ${ }^{10}$ La colocación de angiocatéteres fenestrados \pm masaje compresivo se ha empleado con diversos grados de éxito. El principal inconveniente de este método es la oclusión de angiocatéter debido a su pequeño diámetro. El tiempo para completar la resolución es también mayor en comparación con otros métodos. ${ }^{11} \mathrm{El}$ masaje manual compresivo repetido aumenta la efectividad, pero resulta en un incremento del tiempo de asistencia por personal médico. Los drenajes subcutáneos que utilizan tubos de caucho de tipo Penrose, los drenajes Jackson-Pratt o el drenaje del tórax colocado por técnica de Seldinger se han descrito como efectivos. ${ }^{12,13}$ Algunos de estos métodos han sido asistidos por masaje manual compresivo repetido o succión con presión negativa de bajo nivel. Las incisiones infraclaviculares son un método efectivo para tratar el enfisema subcutáneo extenso, los inconvenientes relacionados con esta técnica citan la necesidad de recambio del apósito (cubierta) de manera regular, riesgo de infec-

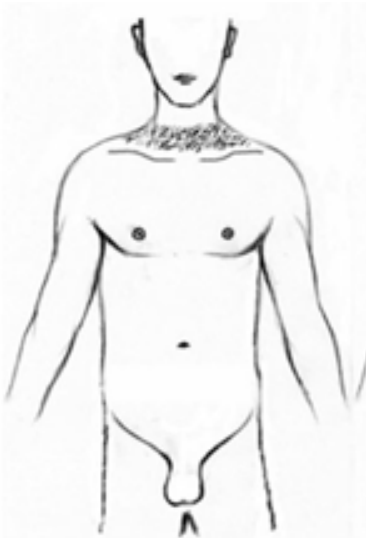

Grado I

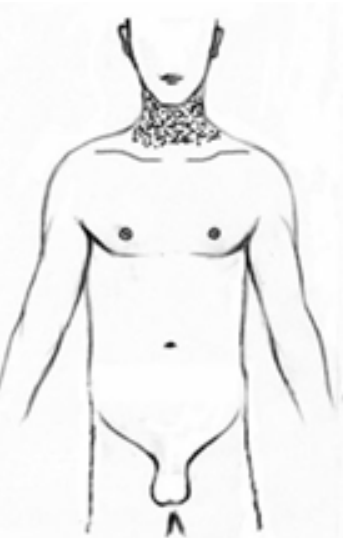

Grado II

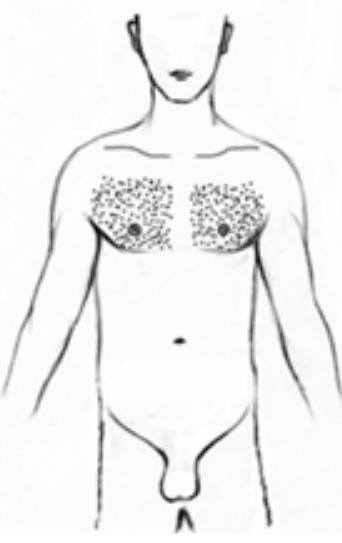

Grado III

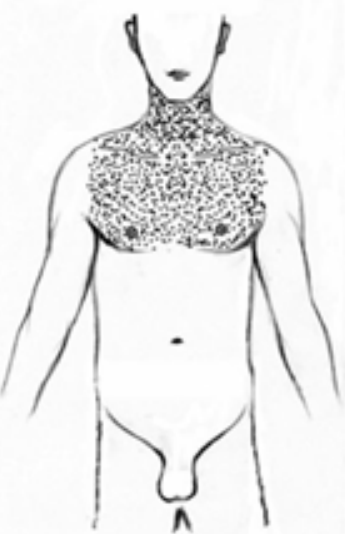

Grado IV

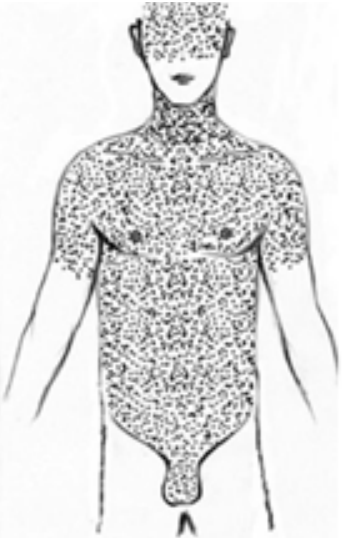

Grado V

Figura 4: Clasificación de la severidad del enfisema subcutáneo. Adaptado de: Aghajanzadeh M, et al. ${ }^{9}$ 
ción, desarrollo de cicatriz desagradable para el paciente y eventualmente el cierre espontáneo. ${ }^{3}$

Byun ${ }^{14}$ demostró en una serie de 10 pacientes con enfisema subcutáneo masivo por ventilación mecánica a presión positiva, que la terapia de cierre asistido por vacío a $-150 \mathrm{mmHg}$ es eficaz. La duración media de la terapia asistida con el sistema de vacío fue de $7.3 \pm 4.8$ días y el número medio de recambios de apósito fue de $1.4 \pm 0.5$ días.

Cerfolio ${ }^{15}$ examinó en un estudio retrospectivo los registros de pacientes sometidos a resección pulmonar $(n=4.023)$. De éstos, 225 desarrollaron enfisema subcutáneo clínicamente significativo. El manejo inicial fue mediante el incremento de la succión aplicada al tubo endopleural. A pesar de esta medida, 85 de los casos presentaron enfisema subcutáneo recalcitrante. La inserción de un tubo torácico adicional a la cabecera se utilizó en 21 de estos pacientes. Los 64 pacientes restantes se sometieron a cirugía torácica videoasistida con identificación y cierre de la fuga. Se insertó un tubo torácico de $28 \mathrm{Fr}$ con una succión de $-40 \mathrm{~cm}$. El enfisema subcutáneo se resolvió en 63 de los 64 casos. Los pacientes con cirugía torácica videoasistida tuvieron estadías hospitalarias significativamente más cortas en comparación con los pacientes sin esta cirugía. Los 85 pacientes en su totalidad fueron dados de alta a casa un día después de la resolución con tubos torácicos unidos a dispositivos portables.

Lloyd y Jankowksi ${ }^{16}$ informaron dos casos de enfisema subcutáneo casi fatal. Mediante la inserción de un catéter fenestrado $28 \mathrm{Fr}$ a cada paciente por vía subcutánea en el quinto espacio intercostal se conectó a succión continua. Ambos pacientes experimentaron notable alivio en una hora. Sólo se observó una leve equimosis en el trayecto de la liposucción.

Beck $^{2}$ describió el primer caso en el que se empleó un microdrenaje al insertar de manera bilateral un angiocatéter fenestrado $14 \mathrm{G}$ por vía subcutánea. A estas cánulas con ayuda de un bisturí se les realizó manualmente fenestraciones. El enfisema subcutáneo quedó resuelto en el día tres postinserción con retiro del drenaje en el día cinco. Los pacientes fueron egresados el día nueve sin complicaciones.

Kelly ${ }^{17}$ describió un caso usando un catéter fenestrado $28 \mathrm{Fr}$ para drenar el enfisema subcutáneo. Se observó una resolución rápida con retiro del drenaje a las 24 horas. El paciente fue egresado del hospital una semana posterior sin complicaciones.

Leo ${ }^{11}$ describió una revisión de 12 pacientes que desarrollaron enfisema subcutáneo y que requirieron microdrenaje. Se aplicaron angiocatéteres fenestrados ayudados por un masaje compresivo administrado de tres a cuatro veces al día. En 11 de los 12 casos el enfisema subcutáneo se resolvió en tres días. Un caso presentó recurrencia del enfisema subcutáneo tras el retiro del drenaje, requiriendo dos días adicionales de microdrenaje. Las heridas se dejaron cicatrizar naturalmente después de la eliminación del drenaje, proporcionando un periodo adicional de 12 a 24 horas de alivio. El autor señaló un riesgo de oclusión del microdrenaje posterior al día tres, requiriendo reemplazo del drenaje.

Srinivas ${ }^{18}$ y Ozdogan ${ }^{19}$ también describieron en estudios independientes la técnica de microdrenaje con masaje compresivo. Ambos lograron una resolución rápida del enfisema subcutáneo en 12 horas sin complicaciones.

Cesaria ${ }^{13}$ revisó 21 casos de pacientes que requirieron drenaje de enfisema subcutáneo. El drenaje se realizó a través de pequeños tubos de caucho tipo Penrose, los cuales fueron insertados subcutáneamente a través de incisiones supraclaviculares. Se requirió del tratamiento por una media de 3.5 días. Todos los casos se resolvieron sin complicaciones.

Matsushita ${ }^{20}$ empleó la misma técnica descrita por Cesaria $^{13}$ con la modificación de aplicar sobre los drenajes bolsas de colostomía para prevenir infecciones. Se resolvió el enfisema en todos los pacientes sin complicaciones.

Sherif y $\mathrm{Ott}^{12}$ describieron un caso en el que utilizaron un drenaje de Jackson-Pratt; drenaje de succión cerrado con depósito de bulbo, que proporcionó la descompresión del enfisema subcutáneo de cara y cuello en tres horas. El drenaje se retiró en el día siete sin complicaciones.

Herlam $^{3}$ examinó el uso de incisiones infraclaviculares sobre la piel y el tejido subcutáneo en cuatro casos. El enfisema subcutáneo se resolvió rápidamente en menos de cuatro días. No se reportaron complicaciones. Otros tres informes de casos utilizaron incisiones infraclaviculares: Dumont y Farag ${ }^{21}$ así como Turnbull ${ }^{22}$ describieron resolución y alivio inmediato de enfisema subcutáneo extenso. Vijayalakshmi y Thinakar $^{23}$ describieron un caso en el cual emplearon incisiones submandibulares con lo cual se resolvió el enfisema subcutáneo en seis días sin complicaciones.

\section{CONCLUSIONES}

El enfisema subcutáneo suele ser una condición benigna y autolimitada que sólo requiere un tratamiento conservador. Las intervenciones analizadas anteriormente son útiles en el contexto de malestar severo, dificultad respiratoria o fuga de aire persistente. Ante la ausencia de cualquier estudio comparativo no es posible escoger definitivamente entre incisión infraclavicular, nueva inserción de drenaje o mayor succión en un drenaje torácico in situ. Se ha demostrado que las tres técnicas descritas proporcionan alivio eficaz del enfisema subcutáneo extenso y grave. 


\section{REFERENCIAS}

1. Rojas M, Rojas P, Toro C, Pinto D, Cifuentes C, Henríquez I, et al. Subcutaneous emphysema after ultrasonic treatment: a case report. Int J Odontostomal. 2009;3:67-70.

2. Beck PL, Heltman SJ, Christopher H. Simple construction of a subcutaneous catheter for treatment of severe subcutaneous emphysema. Chest. 2002;121:647-649.

3. Herlam DB, Landreneau RJ, Ferson PF. Massive spontaneous subcutaneous emphysema. Acute management with infraclavicular "Blow Holes". Chest. 1992;102:503-505.

4. Nair K, Neville E, Rajesh P, Papaliya H. A simple method of pallition for gross subcutaneous surgical emphysema. $J R$ Coll Surg Edinb. 1989;34:163-164.

5. Terada Y, Matsunobe S, Nemoto T, Tsuda T, Shimizu Y. Palliation of severe subcutaneous emphysema with use of trocartype chest tube as a subcutaneous drain. Chest. 1993;103:323.

6. Rydell J, Jennings W. Emergency cervical mediastonotomy for massive mediastinal emphysema. Arch Surg. 1955;70:647-653.

7. Abu-Omar Y, Catarino PA. Progressive subcutaneous emphysema and respiratory arrest. J R Soc Med. 2002;95:90-91.

8. O'Reilly P, Chen HK, Wiseman R. Management of extensive subcutaneous emphysema with a subcutaneous drain. Respirol Case Rep. 2013;1:28-30.

9. Aghajanzadeh M, Dehnado A, Ebrahimi H, Fallah-Karkan M, Khaje-Jahromi S, Amir-Maafi A, et al. Classification and Management of Subcutaneous Emphysema: a 10-year experience. Indian J Surg. 2015;77(Suppl 2):S673-S677.

10. Johnson $\mathrm{CH}$, Lang $\mathrm{SA}$, Bilal $\mathrm{H}$, Rammohan KS. In patients with extensive subcutaneous emphysema, which technique achieves maxima clinical resolution: infraclavicular incisions, subcutaneous drain insertion or suction on in situ chest drain? Interact Cardiovasc Thorac Surg. 2014;18:825-829.

11. Leo F, Solli P, Veronesi G, Spaggiari L, Pastorino U. Efficacy of microdrainage in severe subcutaneous emphysema. Chest. 2002;122:1498-1499.

12. Sherif HM, Ott DA. The use of subcutaneous drains to manage subcutaneous amphysema. Tex Heart Inst J. 1999;26:129-131.

13. Cesaria A, Margaritora S, Porziella V, Granone P. Microdrainage via open technique in severe subcutaneous emphysema. Chest. 2003;123:2161-2162.
14. Byun CS, Choi JH, Hwang JJ, Kim DH, Cho HM, Seok JP Vacuum-assisted closure therapy as an alternative treatment of subcutaneous emphysema. Korean J Thorac Cardiovasc Surg. 2013;46:383-387.

15. Cerfolio RJ, Bryant AS, Maniscalco LM. Management of subcutaneous emphysema after pulmonary resection. Ann Thorac Surg. 2008;85:1759-1765.

16. Lloyd MS, Jankowksi MP. Treatment of life-threatening surgical emphysema with liposuction. Plast Reconstr Surg. 2009;123:77e-78e.

17. Kelly MC, McGuigan JA, Allen RW. Relief of tension subcutaneous emphysema using a large bore subcutaneous drain. Anaesthesia. 1995;50:1077-1079.

18. Srinivas R, Singh N, Agarwal R, Aggaewal AN. Management of extensive subcutaneous emphysema and pneumomediastinum by microdrainage: time for a rethink? Singapore Med J. 2007;48:e323.

19. Ozdogan M, Gurer A, Gokakin AG, Gogkus S, Gomceli I, Aydin R. Treatment of severe subcutaneous emphysema bu fenestrated angiocatheter. Intensive Care Med. 2005;31:168.

20. Matsushita T, Tuan Huynh A, Singh T, Thomson D. Management if life-threatening subcutaneous emphysema using subcutaneous Penrose drains and colostomy bags. Heart Lung Circ. 2007; 16:469-471.

21. Dumont SW, Farag A. Life threatening subcutaneous emphysema. Anaesthesia. 2008;63:212-213.

22. Turnbull DK, Heap MJ. Subcutaneous emphysema and respiratory failure managed with infrclavicular blow holes. Acta Anaesthesiol Scand. 2002;46:227.

23. Vijayalakshmi AM, Thinakar Vel M. Subcutaneous emphysema. Indian Pediatr. 2003;40:1092-1093.

Correspondencia:

José Martin Meza Márquez

E-mail: galeno_78@yahoo.com.mx 